\title{
Minimally invasive lateral corpectomy for thoracolumbar traumatic burst fractures
}

\author{
Adam G. Podet, MD, ${ }^{1}$ Kevin D. Morrow, MD, ${ }^{1}$ Jared M. Robichaux, MD, ${ }^{1}$ \\ Jessica A. Shields, MD, PhD, ${ }^{1}$ Anthony M. DiGiorgio, DO, MHA, ${ }^{1,2}$ and Gabriel C. Tender, MD1
}

${ }^{1}$ Department of Neurosurgery, Louisiana State University Health Sciences Center, New Orleans, Louisiana; and 2Department of Neurological Surgery, University of California, San Francisco, California

OBJECTIVE The need for anterior column reconstruction after thoracolumbar burst fractures remains controversial. Here, the authors present their experience with minimally invasive lateral thoracolumbar corpectomies for traumatic fractures.

METHODS Between 2012 and 2019, 59 patients with 65 thoracolumbar fractures underwent 65 minimally invasive lateral corpectomies (MIS group). This group was compared to 16 patients with single-level thoracolumbar fractures who had undergone open lateral corpectomies with the assistance of general surgery between 2007 and 2011 (open control group). Comparisons of the two groups were made with regard to operative time, estimated blood loss, time to ambulation, and fusion rates at 1 year postoperatively. The authors further analyzed the MIS group with regard to injury mechanism, fracture characteristics, neurological outcome, and complications.

RESULTS Patients in the MIS group had a significantly shorter mean operative time $(228.3 \pm 27.9$ vs $255.6 \pm 34.1$ minutes, $p=0.001)$ and significantly shorter mean time to ambulation after surgery (1.8 \pm 1.1 vs $5.0 \pm 0.8$ days, $p<0.001)$ than the open corpectomy group. Mean estimated blood loss did not differ significantly between the two groups, though the MIS group did trend toward a lower mean blood loss. There was no significant difference in fusion status at 1 year between the MIS and open groups; however, this comparison was limited by poor follow-up, with only 32 of 59 patients $(54.2 \%)$ in the MIS group and 8 of $16(50 \%)$ in the open group having available imaging at 1 year. Complications in the MIS group included 1 screw misplacement requiring revision, 2 postoperative femoral neuropathies (one of which improved), 1 return to surgery for inadequate posterior decompression, 4 pneumothoraces requiring chest tube placement, and 1 posterior wound infection. The rate of revision surgery for the failure of fusion in the MIS group was $1.7 \%$ (1 of 59 patients).

CONCLUSIONS The minimally invasive lateral thoracolumbar corpectomy approach for traumatic fractures appears to be relatively safe and may result in shorter operative times and quicker mobilization as compared to those with open techniques. This should be considered as a treatment option for thoracolumbar spine fractures.

https://thejns.org/doi/abs/10.3171/2020.6.FOCUS20366

KEYWORDS thoracolumbar burst fractures; lateral lumbar fusion; lateral corpectomy; thoracolumbar corpectomy; spinal cord injury; traumatic spine injury

I NDICATIONS for minimally invasive (MI) approaches in spine surgery continue to evolve. The MI lateral approach to the thoracolumbar spine has become an increasingly popular technique for achieving interbody fusion since it was first described. ${ }^{1}$ Proponents of the lateral approach claim reduced tissue trauma, improved pain scores, earlier mobilization, increased rates of arthrodesis, and greater deformity correction..$^{2-7}$

Data supporting lateral access approaches to the tho- racolumbar spine for degenerative pathology are robust. ${ }^{3,8}$ More recently, data supporting the lateral approach for select postinfectious and neoplastic spinal pathology have expanded the indications for this technique. ${ }^{9-11}$ Consequently, the use of MI lateral spinal fusion has become a prevalent tool in the surgical management of degenerative, neoplastic, and postinfectious spinal pathology.

However, data on the MI lateral access approaches in the context of trauma are relatively scarce, with only small

ABBREVIATIONS AIS = American Spinal Cord Injury Association (ASIA) Impairment Scale; EBL = estimated blood loss; MEP = motor evoked potential; MI = minimally invasive; PLC = posterior ligamentous complex; TLICS = thoracolumbar injury classification and severity.

SUBMITTED April 30, 2020. ACCEPTED June 10, 2020.

INCLUDE WHEN CITING DOI: 10.3171/2020.6.FOCUS20366. 
case reports evaluating heterogeneous patient populations, fracture morphologies, and surgical techniques. ${ }^{12,13}$ To our knowledge, few studies have examined the role of the MI lateral approach accompanied by anterior column reconstruction (MI lateral corpectomy) in a trauma population. ${ }^{14-16}$

The indications for surgery in thoracolumbar trauma have evolved with improvements in diagnostic testing. The thoracolumbar injury classification and severity (TLICS) system characterizes fracture morphology, posterior ligamentous complex (PLC) integrity, and neurological status. ${ }^{17-19}$ Patients with a TLICS score $\geq 5$ are candidates for surgery. In patients with burst fractures, thoracolumbar corpectomies can be performed via a posterolateral or anterolateral (transthoracic or retroperitoneal) approach. ${ }^{20-22}$ The need for anterior column reconstruction after thoracolumbar burst fractures remains controversial, ${ }^{23,24}$ and little prospective data exist. However, the anterolateral approach may offer better access to fracture fragments encroaching the spinal canal, while also facilitating larger implant insertion.

Our hospital is a level I trauma center in the southern United States. Since 2012, all thoracolumbar burst fractures meeting surgical criteria have been managed with anterior column reconstruction via an MI lateral retroperitoneal or retropleural approach. We compared the basic perioperative results in these patients with those in a control group that had undergone open lateral corpectomies from 2007 to 2011. We further analyzed other parameters only available in the MI group. To our knowledge, this is the largest series of thoracolumbar burst fractures treated by MI lateral corpectomy.

\section{Methods \\ Patient Population}

This is a retrospective analysis of prospectively collected data. Between January 2012 and September 2019, 59 patients with 65 thoracolumbar fractures underwent $65 \mathrm{MI}$ lateral corpectomies (MIS group) at our institution. This group was compared to patients with single-level thoracolumbar fractures who had undergone open lateral corpectomies with the assistance of general surgery between January 2007 and December 2011 (open control group). The following parameters were collected routinely and were available for both groups: fracture level, procedure time, estimated blood loss (EBL), time to ambulation (in patients without other factors precluding them from ambulating), perioperative complications, and fusion status at 1 year. Other data available for only the MIS group included injury mechanism, preoperative and postoperative neurological status, time to surgery, late complications ( $>2$ months postsurgical), and the need for any revision surgery.

\section{Inclusion and Exclusion Criteria}

Patients who had undergone MI lateral corpectomy performed by the senior author (G.C.T.) for the treatment of thoracolumbar burst fractures between January 2012 and September 2019 were included in the MIS group. Thirteen patients who had undergone delayed operative intervention after failed conservative management were also included in this group. Patients who had undergone open lateral corpectomy performed by the senior author assisted by general surgery prior to 2012 were included in the open control group. Patients were excluded if fractures were deemed pathological, or if patients were under the age of 18 years at the time of treatment.

\section{Operative Indications}

All patients presenting to our center with comminuted thoracolumbar vertebral body fractures of T6-L4 with PLC disruption were considered for lateral corpectomy (TLICS score $\geq 5$ ). Indications for lateral corpectomy were burst fractures with loss of vertebral body height $>50 \%$, kyphotic angulation $>30^{\circ}$, or a young patient in whom a long-segment fusion would be undesirable. Anatomical considerations involved an anterior trajectory of the femoral nerve on MRI (L3 and L4 fractures) and iliac crest height (L4 fractures). Patients with unfavorable anatomy were managed with an alternative surgical strategy and were not included in this study.

The decision for supplemental fixation (lateral plate, posterior percutaneous, or open pedicle screw instrumentation) was made based on the following algorithm (Fig. $1)$. Patients with severe canal compression $(\geq 50 \%)$ were recommended for posterior open decompression, regardless of neurological status. In patients with $<50 \%$ canal stenosis with associated neurological deficits, posterior open decompression was also recommended. According to this algorithm, patients who required posterior open decompression received supplementation with conventional open pedicle screw instrumentation one level above and one level below the corpectomy level(s). In patients with multiple unstable vertebral body fractures, we increased the level of instrumented vertebrae to ensure adequate stabilization. Fractures that did not require decompression were supplemented with percutaneous instrumentation one level above and one level below the index vertebra. Only fractures without PLC disruption (as in low TLICS fractures that failed conservative management) were supplemented with a lateral plate alone.

\section{MIS Group: Surgical Technique}

This technique has been described elsewhere, ${ }^{14}$ and here we briefly present our surgical steps. All procedures are performed with the patient under general anesthesia with intraoperative neurophysiological monitoring consisting of continuous monitoring of somatosensory evoked potentials and frequent monitoring of motor evoked potentials (MEPs). The patients undergo standard intubation (in our experience, there is no need for unilateral lung deflation, though this technique can still be employed at the surgeon's discretion). The patient is placed in a right lateral decubitus position (the left lateral decubitus can be used, if the anatomy is preferable) and taped to the operating table.,14 The index vertebral body is marked on the skin using fluoroscopy. Intraoperative CT and spinal navigation were not used for the cases in this series.

A 7- to 8-cm skin incision is centered over the fractured segment in line with the underlying rib (or parallel to the iliac crest for an L4 corpectomy). The rib is detached from 


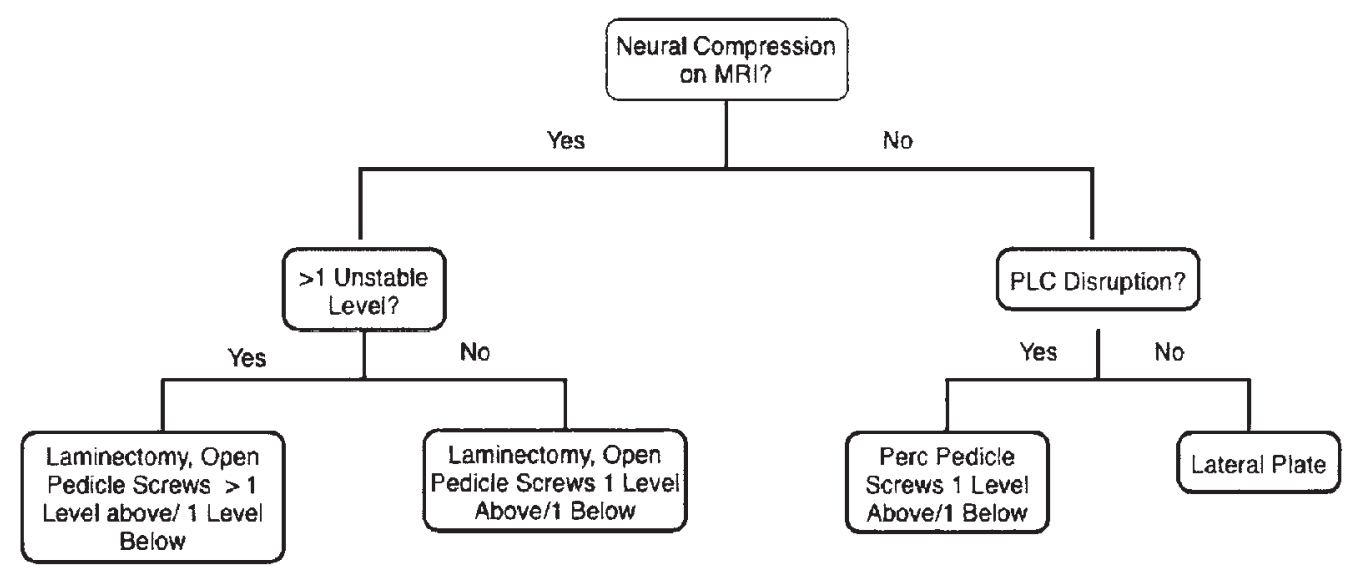

FIG. 1. Instrumentation decision algorithm. Perc = percutaneous.

the intercostal muscles and transected at the edges of the exposure. Using finger dissection, the parietal pleura or retroperitoneal fat is detached from the remaining portion of the proximal rib, as well as the two adjacent ribs. The tubular dilator is placed at the junction of the rib and the vertebral body. Under lateral fluoroscopy, the three-blade retractor is positioned with the posterior blade in line with the posterior wall of the vertebral body. The remainder of the operation is performed under the microscope.

In the lumbar spine, the psoas muscle is dissected to expose the discs above and below the index vertebra. The segmental vessel is exposed and temporarily occluded while monitoring for any change in MEPs suggestive of Adamkiewicz artery ischemia. If any reduction in MEPs is detected, the vessel cannot be sacrificed. ${ }^{25}$ Otherwise, the vessel is transected, and the lateral aspect of the vertebral body is exposed.

A standard technique is used to remove the adjacent discs. ${ }^{1}$ We perform the corpectomy before decompression of the spinal canal to ensure optimal placement of the cage. The corpectomy must be sufficiently wide to accommodate the implant without displacing fracture fragments posteriorly into the canal. We perform the corpectomy with osteotomes to maximize speed and minimize bleeding. We tamponade the exposed bone with hemostatic agent while preparing the endplates. An expandable cage is then inserted between two protective sliding blades under anteroposterior fluoroscopy and expanded to the desired height.

If needed, decompression of the spinal canal is performed after placement of the cage. The retractor is angled from anterior to posterior, and the fractured posterior border of the vertebral body is carefully pushed anteriorly using a small straight curette. Epidural bleeding is easily controlled with Gelfoam and gentle pressure. Full decompression is confirmed by reexpansion of the dural sac anteriorly, into its usual anatomical position.

A lateral plate with four screws can be attached to the vertebral bodies above and below the fracture. Alternatively, the patient can undergo posterior pedicle screw-rod fixation, typically one level above and below the fracture. Though some surgeons prefer to perform posterior fixation in the lateral position, our practice is to carefully reposition the patient prone.
Though we attempt to maintain the integrity of the parietal pleura, violation is sometimes unavoidable. In these cases, a primary closure of the pleura is unnecessary. Before removing the retractor, a Jackson-Pratt 7-mm flat drain is tunneled to the lateral aspect of the corpectomy cage and left to suction. The drain serves both to evacuate postoperative bleeding and to prevent a tension pneumothorax. The intercostal muscle layer should be closed in a watertight fashion, especially if the parietal pleura has been violated. If the visceral pleura (i.e., the lung parenchyma) is injured, a formal chest tube is placed prior to extubation to prevent tension pneumothorax. After all cases, we routinely obtain a postoperative chest radiograph and closely monitor the oxygen saturations to monitor for the development of a tension pneumothorax.

\section{Open Group: Surgical Technique}

Open corpectomies were performed with general surgery assistance. For lumbar fractures, this required a 30to $40-\mathrm{cm}$ incision and extensive lateral abdominal muscle dissection. The psoas muscle was released from its anterior insertion and retracted en bloc over three levels (one above and one below the index level). We placed screws in the vertebral bodies above and below the fracture and used the designated spreader to insert a static cage (expandable technology was not available at that time). The screws were then connected by lateral rods.

For thoracic fractures, the general/thoracic surgeon requested dual-lumen intubation and unilateral lung deflation for the duration of the surgery. After completion of the corpectomy and fusion, a chest tube was routinely inserted in all patients and weaned over several days.

\section{Radiographic Fusion Criteria}

CT images obtained at 1 year postoperatively with evidence of bridging trabeculae between the vertebral bodies or across the facet joints were used as radiographic evidence of fusion. In some cases, bridging trabeculae can be seen through the cage, but usually the metal artifact makes this impossible. Alternatively, flexion/extension radiographs obtained at 1 year postoperatively without evidence of abnormal motion or hardware failure were 
TABLE 1. Characteristics of the MIS group

\begin{tabular}{|c|c|}
\hline Variable & Value \\
\hline No. of patients & 59 \\
\hline No. of burst fractures & 65 \\
\hline Male sex, no. (\%) & $41(69.5)$ \\
\hline Mean age in yrs (range) & $41.2(18-65)$ \\
\hline Polytrauma, no. (\%) & $34(57.6)$ \\
\hline \multicolumn{2}{|l|}{ Mechanism of injury, no. (\%) } \\
\hline Fall from height & $29(49.2)$ \\
\hline MVC & $25(42.4)$ \\
\hline Recreational vehicle accident & $2(3.4)$ \\
\hline Pedestrian vs vehicle & $2(3.4)$ \\
\hline Helicopter & $1(1.7)$ \\
\hline Burst fractures, no. (\%) & 65 \\
\hline $\mathrm{T} 7$ & $2(3.1)$ \\
\hline T8 & $4(6.2)$ \\
\hline T9 & $3(4.6)$ \\
\hline T10 & $1(1.5)$ \\
\hline T11 & $3(4.6)$ \\
\hline $\mathrm{T} 12$ & $8(12.3)$ \\
\hline L1 & $24(36.9)$ \\
\hline L2 & $7(10.8)$ \\
\hline L3 & $8(12.3)$ \\
\hline L4 & $5(7.7)$ \\
\hline Preop neurological injuries, no. (\%) & $16(27.1)$ \\
\hline AIS grade $A$ & $6(10.2)$ \\
\hline AIS grade $B$ & $0(0)$ \\
\hline AIS grade $\mathrm{C}$ & $1(1.7)$ \\
\hline AIS grade $D$ & $0(0)$ \\
\hline Cauda equina & $5(8.5)$ \\
\hline Neurogenic bladder & $3(5.1)$ \\
\hline Isolated sensory & $1(1.7)$ \\
\hline Periop complications, no. (\%) & $9(15.3)$ \\
\hline MIS corpectomy-related & $6(10.2)$ \\
\hline Pneumothorax & $4(6.8)$ \\
\hline Femoral neuropathy & $2(3.4)$ \\
\hline Posterior wound infection & $1(1.7)$ \\
\hline Inadequate decompression & $1(1.7)$ \\
\hline Misplaced pedicle screw & $1(1.7)$ \\
\hline
\end{tabular}

$\mathrm{MVC}=$ motor vehicle collision

considered to demonstrate radiographic fusion. Any radiographic evidence of graft resorption, hardware failure, or abnormal dynamic motion was considered evidence of pseudarthrosis.

\section{Statistical Analysis}

Statistical analysis was performed using an independent-samples t-test to compare the MIS and open groups with regard to each variable (procedure time, EBL, time to ambulation, and fusion at 1 year). Levene's test for equality of variances was used to ensure that no significant variability existed between the groups as the sample sizes dif-
TABLE 2. Comparison of MIS and open lateral corpectomy perioperative outcomes

\begin{tabular}{lccc}
\hline \multicolumn{1}{c}{ Variable } & MIS Group & Open Group & p Value \\
\hline No. of patients & 59 & 16 & \\
\hline Male sex & $41(69.5)$ & $10(62.5)$ & \\
\hline Mean age in yrs (range) & $41.2(18-65)$ & $39.5(20-59)$ & \\
\hline Single-level fracture & 53 & 16 & \\
\hline Multilevel fractures & 6 & 0 & \\
\hline Level treated & 65 & 16 & \\
T7 & $2(3.1)$ & 0 & \\
T8 & $4(6.2)$ & 0 & \\
T9 & $3(4.6)$ & 0 & \\
T10 & $1(1.5)$ & $1(6.3)$ & \\
T11 & $3(4.6)$ & $1(6.3)$ & \\
T12 & $8(12.3)$ & $4(25.0)$ & \\
L1 & $24(36.9)$ & $4(25.0)$ & \\
L2 & $7(10.8)$ & $2(12.5)$ & \\
L3 & $8(12.3)$ & $2(12.5)$ & \\
L4 & $5(7.7)$ & $2(12.5)$ & \\
\hline OR time in mins (SD) & $228.3(27.9)$ & $255.6(34.1)$ & 0.001 \\
\hline Time to ambulation in days & $1.8(1.1)$ & $5.0(0.8)$ & $<0.001$ \\
(SD) & & & \\
\hline EBL in ml (SD) & $2211.9(409.4)$ & $2406.2(466.8)$ & 0.106 \\
\hline 1-yr FU & $32(54.2)$ & $8(50.0)$ & \\
\hline Fused at 1 yr $(n=32)$ & $29(90.6)$ & $8(100)$ & 0.4 \\
\hline Pseudarthrosis $(n=32)$ & $1(3.1)$ & 0 & \\
\hline Subsidence $(n=32)$ & $2(6.3)$ & 0 & \\
\hline FU $=$ folow- $n=n+m b e r$ & & & \\
\hline
\end{tabular}

$\mathrm{FU}=$ follow-up; $\mathrm{n}=$ number.

Values are presented as the number of patients (\%), unless otherwise indicated. Boldface type indicates statistical significance.

fered. All $\mathrm{p}$ values were $>0.16$, suggesting that equal variances could be assumed. Data were analyzed using SPSS (IBM Corp.). A p value $<0.05$ was considered statistically significant.

\section{Results}

\section{Patient Demographics}

In the MIS group, 59 patients had sustained 65 thoracolumbar burst fractures and underwent $65 \mathrm{MI}$ lateral corpectomy procedures. The patients were predominantly male (41 of 59 patients [69.5\%]) and had a mean age of 41.2 years (range 18-65 years; Table 1). In the open group, 16 patients had sustained 16 thoracolumbar burst fractures and underwent 16 open corpectomy procedures.

\section{Open Versus MIS Comparison}

Comparisons were made with regard to mean procedure time, EBL, time to ambulation, and fusion status at 1 year (Table 2). Patients who underwent the MI lateral corpectomy had a significantly shorter procedure time (228.3 \pm 27.9 vs $255.6 \pm 34.1$ minutes, $p=0.001$; Fig. 2 A) and significantly shorter time to ambulation after surgery $(1.8 \pm$ 1.1 vs $5.0 \pm 0.8$ days, $\mathrm{p}<0.001)$ than the open corpectomy group (Fig. 2C). EBL did not differ significantly between the two groups, though the MIS group did tend toward a 


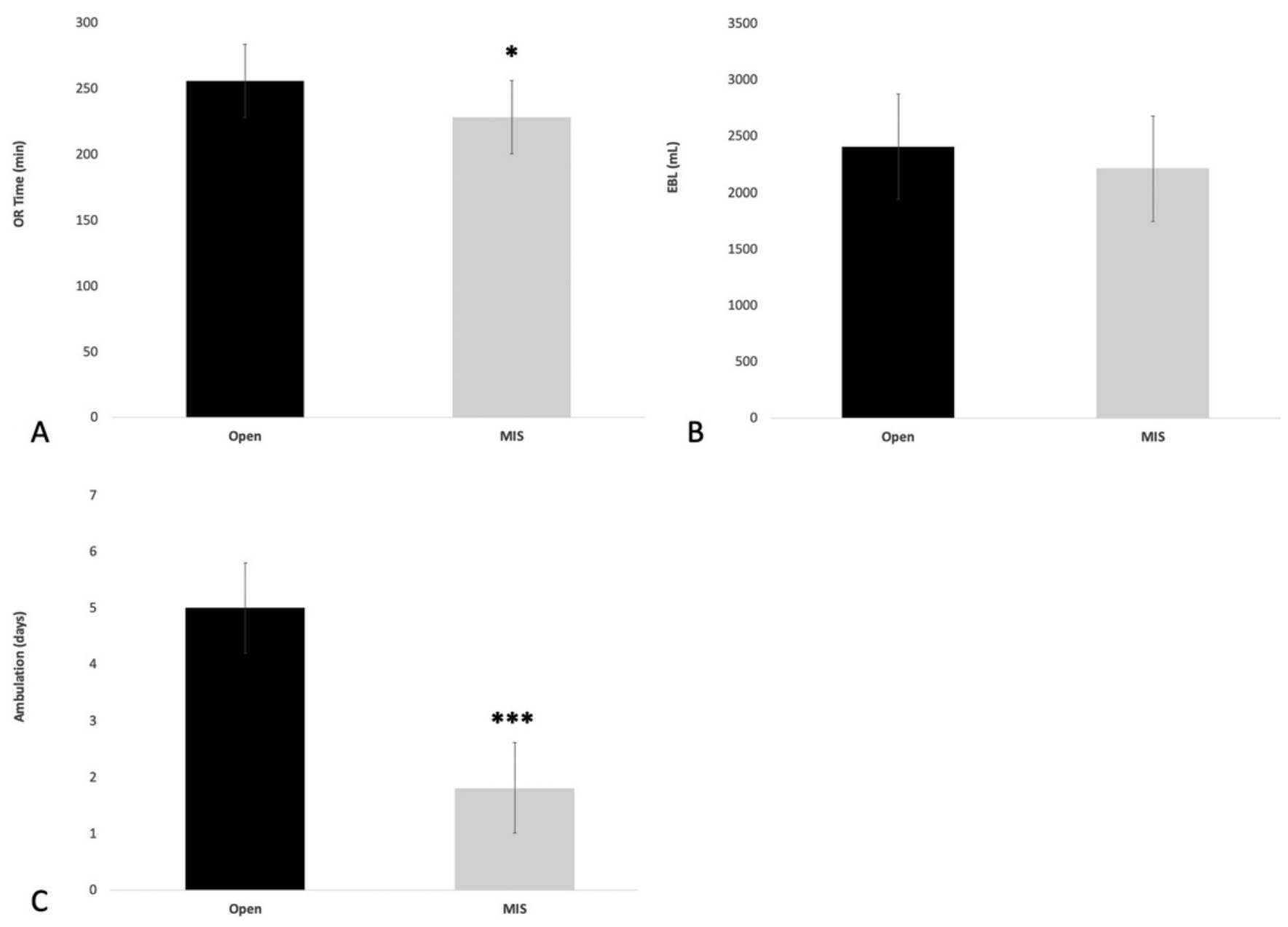

FIG. 2. A: The operating room (OR) time was significantly lower in the MIS group than in the open group. B: There was no significant difference in EBL between the MIS and open groups. C: There was a significantly shorter time to ambulation in the MIS group than in the open group. ${ }^{*} p=0.001 ;{ }^{* * *} p<0.001$

lower mean blood loss $(2211.9 \pm 409.4$ vs $2406.2 \pm 466.8$ $\mathrm{ml}, \mathrm{t}=1.63, \mathrm{p}=0.106 ;$ Fig. 2B).

There was no significant difference in fusion status at 1 year between the MIS and open groups $(t=0.9, p=0.4$; Table 2). However, this comparison was limited by poor follow-up. Only half of the open group had radiographic follow-up, with no patients showing evidence of fusion failure. Thirty-two of the 59 MIS patients (54.2\%) were available for radiographic follow-up at 1 year. The average follow-up was 13.8 months. Twenty-nine of these 32 patients $(90.6 \%)$ fulfilled the criteria for radiographic fusion. One patient with posterior fusion sustained pseudarthrosis, while 1 patient with a lateral plate sustained pseudarthrosis. Two of the 32 MIS patients (6.3\%) had cage subsidence, with neither patient requiring revision surgery. The overall rate of revision surgery for the failure of fusion in the MIS group was 1.7\% (1 of 59 patients).

\section{Results for the MIS Group}

Mechanism of Injury

The most common mechanism of injury was a fall from height (29 of 59 [49.2\%]), followed by a motor ve- hicle collision (25 of 59 [42.4\%]). Other infrequent injury mechanisms included recreational vehicle accident (2 of 59 [3.4\%]), helicopter crash (1 of 59 [1.7\%]), and pedestrian versus vehicle ( 2 of 59 [3.4\%]). The majority of patients sustained polytrauma injuries (34 of 59 [57.6\%]; Table 1).

\section{Spine Injury Characteristics}

Thoracolumbar burst fractures treated with the MI lateral corpectomy ranged from levels T7 to L4. The most commonly treated vertebra was L1, which accounted for 24 of 65 treated vertebrae (36.9\%), followed by L3 ( 8 of 65 [12.3\%]) and T12 (8 of 65 [12.3\%]; Table 1). Six of 59 patients $(10.2 \%)$ underwent two-level corpectomy accounting for 12 of 65 treated fractures (18.5\%). All fractures were comminuted burst fractures with PLC disruption.

Sixteen of the 59 patients (27.1\%) presented with neurological deficits attributed to the index spinal injury. Six of the 59 patients (10.2\%) sustained American Spinal Cord Injury Association (ASIA) Impairment Scale (AIS) grade A spinal cord injuries at the time of presentation. One patient $(1.7 \%)$ sustained an AIS grade C injury. Five of the 59 patients $(8.5 \%)$ presented with mixed motor and sensory 


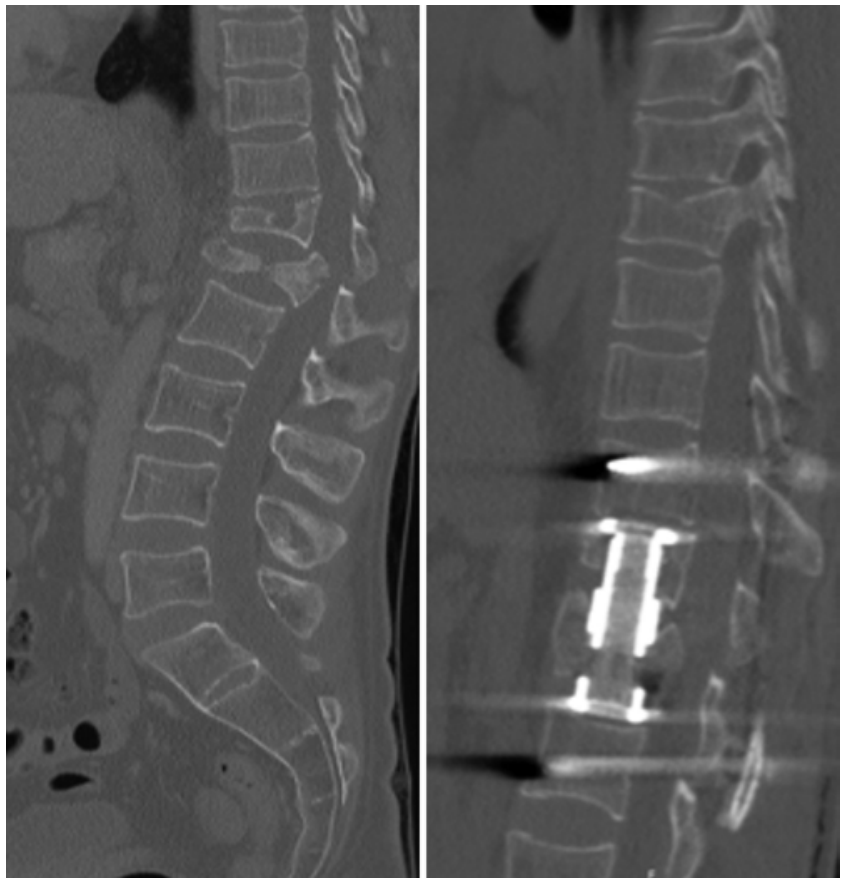

FIG. 3. CT depicting tandem T12-L1 burst fractures before (left) and after (right) two-level MI lateral corpectomy following the failure of conservative management.

deficits secondary to cauda equina injury. Three of the 59 patients $(5.1 \%)$ sustained isolated neurogenic bladder injuries in the absence of motor or sensory deficits. One patient $(1.7 \%)$ presented with an isolated sensory deficit (Table 1).

\section{Procedural Characteristics}

The majority of patients (53 of 59 [89.8\%]) underwent single-level thoracolumbar corpectomy, accounting for 53 of the 65 thoracolumbar burst fractures $(81.5 \%)$ treated. Six of the 59 patients $(10.2 \%$, or 12 of 65 fractures [18.5\%]) underwent two-level corpectomy to manage tandem burst fractures (Fig. 3). The vast majority of patients (53 of 59 [89.8\%]) received supplementation with posterior pedicle screw fixation to include the level above and below the index vertebra(e) (Fig. 4). Posterior instrumentation was deemed unnecessary based on injury morphology in only 6 of 59 patients (10.2\%), who instead received supplementation with a lateral plate.

The time to surgery was variable, depending primarily on obtaining clinical stability, but the average time was 4.3 days (range $0-42$ days). Thirteen patients were treated with delayed surgery in the follow-up. These patients had uniformly sustained relatively minor fractures (TLICS score $\leq 4$ ), which failed conservative management (Fig. 5). In 1 patient with a TLICS score of 5, a trial of conservative care was attempted and failed, although surgical intervention had been recommended.

\section{Perioperative Complications}

There were 9 total perioperative complications (15.3\%) in the MIS group (Table 1). Three of these complications were unrelated to the corpectomy portion of the proce-
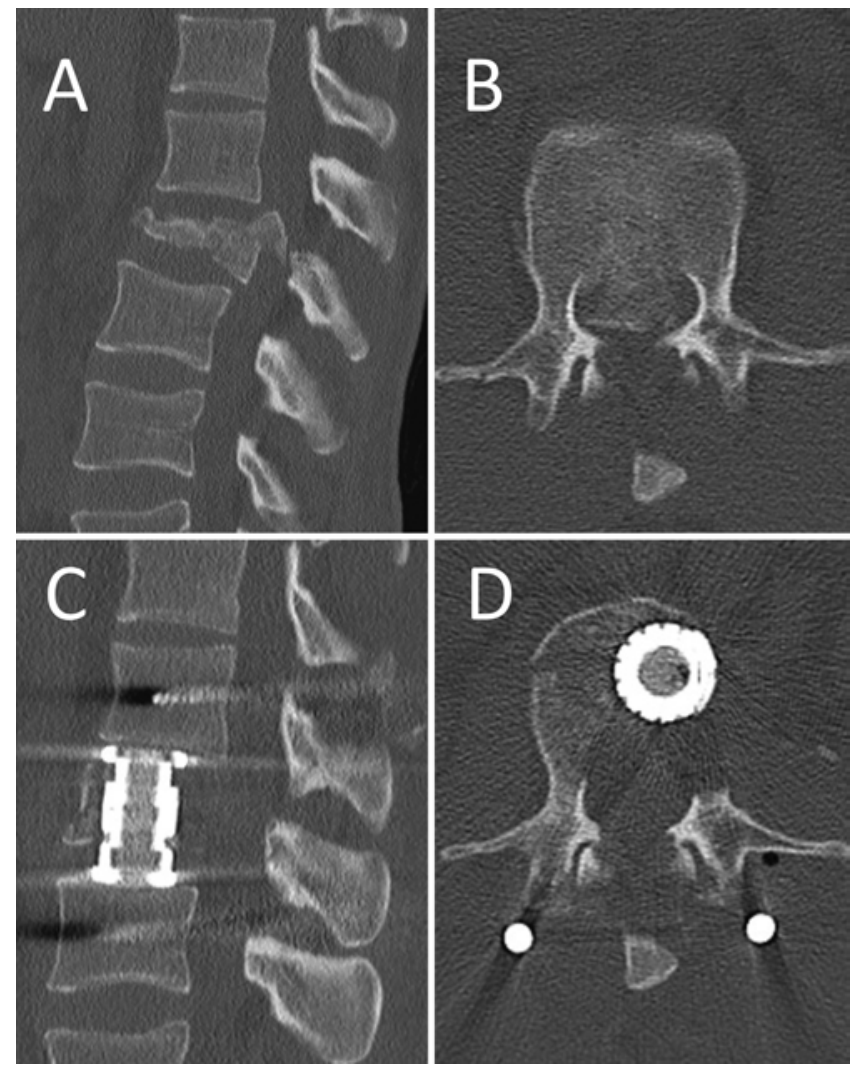

FIG. 4. Preoperative sagittal (A) and axial (B) CT depicting an L2 comminuted burst fracture with $>50 \%$ loss of vertebral body height and severe retropulsion of displaced fracture fragments with obliteration of the spinal canal. Postoperative sagittal (C) and axial (D) CT of the same fracture following anterior translation of retropulsed fragments to decompress the spinal canal. A corpectomy cage has been placed, with posterior fixation extending one level above and one level below the fractured vertebra.

dure: 1 posterior wound infection, 1 misplaced pedicle screw, and 1 inadequate posterior decompression with postoperative neurological deterioration. Perioperative complications directly related to the MI lateral corpectomy included 4 pneumothoraces requiring chest tube placement (4 of 59 [6.8\%]) and 2 femoral neuropathies (2 of 59 [3.4\%]) causing leg weakness and paresthesia (one of which resolved at follow-up).

\section{Neurological Outcome}

Of the 16 patients with a neurological deficit in the MIS group, 9 (56.3\%) recovered at least partially. All 6 AIS grade A spinal cord injuries (100\%) maintained this classification at the last follow-up. One patient with an L1 AIS grade $\mathrm{C}$ spinal cord injury at presentation improved to AIS grade D. Two of the 3 patients with isolated neurogenic bladder had complete improvement at the follow-up. Three of 5 patients with mixed sensory and motor deficits due to cauda equina injury had complete neurological recovery at the last follow-up, while one had a complete motor recovery with persistent sensory deficit and one had no neurological recovery. Two patients (3.4\%) experienced neurological deterioration that persisted at follow-up. Both 


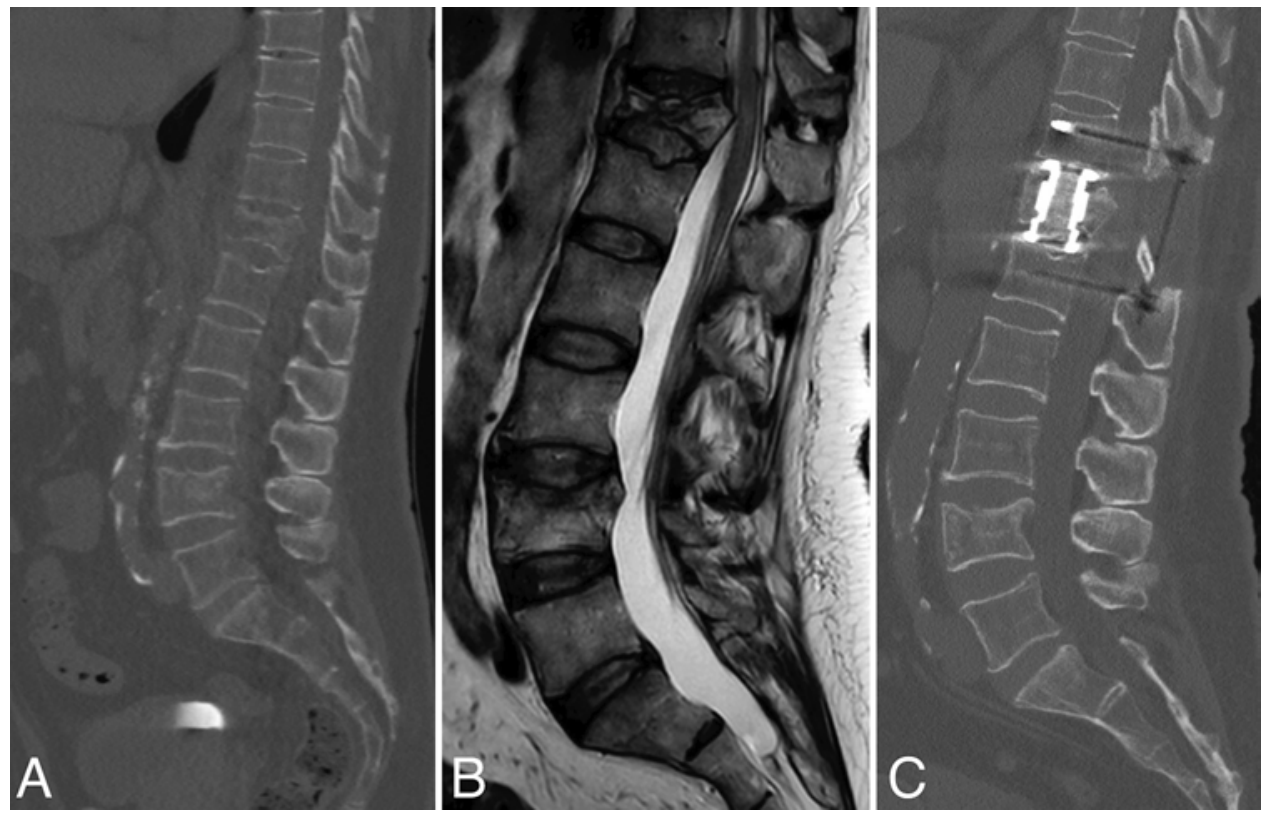

FIG. 5. Sagittal CT (A) depicting a two-column T12 burst fracture with $<50 \%$ loss of vertebral body height and minimal retropulsion, with additional L1 and L4 mild compression deformities (initially managed conservatively). Six-week follow-up MR image (B) showing progression of the T12 burst fracture, now with 60\% vertebral body collapse and significant retropulsion causing central canal stenosis. Postoperative CT (C) demonstrating T12 MI lateral corpectomy, laminectomy, and T11-L1 short-segment fusion.

injuries were consistent with femoral neuropathy, one of which improved in severity.

\section{Fusion Outcome and Follow-Up}

Thirty-two of the 59 patients (54.2\%) had follow-up CT or flexion/extension radiographs at 1 year postoperatively. Twenty-nine of these 32 patients $(90.6 \%)$ fulfilled the criteria for radiographic fusion. One of the 32 patients (3.1\%) sustained pseudarthrosis and required revision posterior instrumentation after initial lateral plating. One of the 6 patients $(16.7 \%)$ who had received supplementation with a lateral plate had pseudarthrosis. Two patients $(6.3 \%)$ had cage subsidence, with neither patient requiring revision surgery. The overall rate of revision surgery for failure of fusion was $1.7 \%$ ( 1 of 59 patients), with the lone patient having a lateral plate. Average follow-up was 13.8 months. Twenty-seven of the 59 patients $(45.8 \%)$ were lost to follow-up before 1 year postoperatively.

\section{Discussion}

The MI lateral lumbar corpectomy technique was a natural extension of the extreme lateral interbody fusion techniques. ${ }^{14}$ The advantage of the MI lateral transpsoas lumbar corpectomy technique is that the psoas muscle dissection, cage insertion, and canal decompression can be performed via a small skin incision, whereas the open technique requires a large lateral abdominal wall dissection to allow for exposure of the anterior insertion of the psoas muscle and straight lateral insertion of the interbody implant (Fig. 6). Similarly, the MI thoracic corpectomy techniques proved advantageous, since the small skin incision and limited single-rib resection also allowed for standard intubation without lung deflation.
The literature has not reached a clear consensus regarding the need for anterior column reconstruction after thoracolumbar burst fractures..$^{23,24}$ The theoretical benefit of anterior column reconstruction is that a shorter segment can be fused. Additionally, a corpectomy allows anterior canal decompression by direct removal of fracture fragments encroaching the spinal canal (Fig. 4). Moreover, the anterior approach may offer better sagittal realignment. Most of the studies comparing anterior column recon-
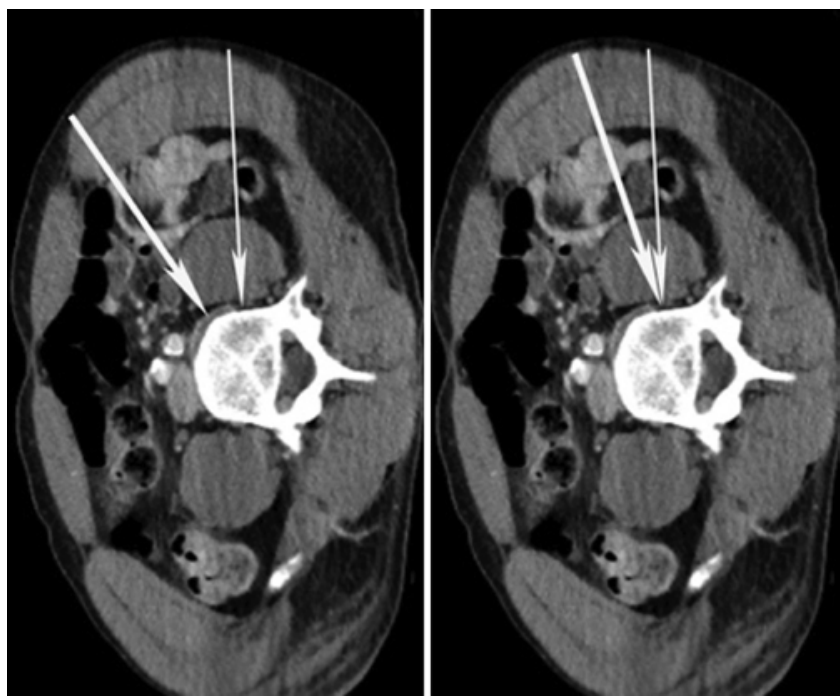

FIG. 6. CT depicting the psoas muscle dissection (thick arrows) and cage insertion (thin arrows) directions in open (left) versus MI (right) techniques. The skin incision and lateral abdominal wall dissection are decreased in the latter approach. 
struction to purely posterior approaches were done with open corpectomies. MI techniques have shown benefits over open corpectomies, both in our data here and in prior studies. ${ }^{11,14-16,26}$ Thus, we believe the addition of anterior column reconstruction in the management of burst fracture, using an MI technique, has clear benefit to the patient.

We were able to obtain solid arthrodesis with relatively few complications, while utilizing short-segment fusions. Using a purely posterior approach for these fractures would involve the typical "two above, two below" algorithm. While this is adequate in many cases, a shorter-segment fusion has benefits. Short-segment fusions are desirable in younger patients, as the transfer of biomechanical stressors will accelerate degeneration in adjacent levels. ${ }^{27}$ Additionally, the correction of posttraumatic kyphotic deformity is augmented with the anterior approach.

In our practice, anterior decompression of the spinal canal is performed when posteriorly displaced fragments result in neurological deficit. In these cases, we prefer to complete the anterior corpectomy and implant insertion first. This facilitates optimal placement of the interbody cage and anterior translation of the fracture fragments (Fig. 4). Concern for posterior displacement of the fracture fragments during cage insertion is mitigated by performing a wide corpectomy to accommodate the implant.

Our algorithm for posterior instrumentation (Fig. 1) is reliably used to standardize our decision-making. Only 1 patient required a revision surgery for pseudarthrosis. Notably, this was in a patient who had received a lateral plate, suggesting that this is not always an adequate stabilization technique in trauma. Admittedly, our follow-up rate is not optimal, but this is a common problem with trauma populations. Our remaining complication rates are consistent with published reports in lateral MI surgery for degenerative or other pathologies. ${ }^{28}$

Notably, data pertaining to the demographic and surgical characteristics of our open control group were incomplete. Therefore, we must acknowledge the possibility that baseline differences may account for the benefits we detected in the MIS group. Still, patients included in this study were obtained from the same trauma registry and managed using the same treatment algorithm. Therefore, these data do not definitively answer any questions regarding the benefits of MI anterior column reconstruction versus posterior-only approaches. However, we believe our series provides evidence that the MI lateral corpectomy has a place in the treatment of thoracolumbar burst fractures. At a minimum, our series shows that MI corpectomies are safe and effective in the trauma population.

As stated previously, a major limitation of our study is the poor follow-up rate. Outside of well-funded registries with resources to ensure follow-up for trauma patients, few centers will have better follow-up rates in these patients. Other limitations include the retrospective nature of our review and the inherent biases within. All cases were performed by the senior author; thus, benefits in the MIS group could be the result of longitudinal effects such as improved surgical efficiency and technique. While these limitations are significant, we believe our series shows the promise of the MI corpectomy technique in treating these challenging fractures.

\section{Conclusions}

The MI lateral thoracolumbar corpectomy approach for traumatic fractures appears safe. Compared to open techniques, this approach results in shorter operative times and earlier mobilization and should be considered in the treatment of traumatic burst fractures in the thoracolumbar spine.

\section{References}

1. Ozgur BM, Aryan HE, Pimenta L, Taylor WR. Extreme Lateral Interbody Fusion (XLIF): a novel surgical technique for anterior lumbar interbody fusion. Spine J. 2006;6(4):435-443.

2. Acosta FL, Liu J, Slimack N, et al. Changes in coronal and sagittal plane alignment following minimally invasive direct lateral interbody fusion for the treatment of degenerative lumbar disease in adults: a radiographic study. J Neurosurg Spine. 2011;15(1):92-96.

3. Anand N, Rosemann R, Khalsa B, Baron EM. Mid-term to long-term clinical and functional outcomes of minimally invasive correction and fusion for adults with scoliosis. Neurosurg Focus. 2010;28(3):E6.

4. Berjano P, Balsano M, Buric J, et al. Direct lateral access lumbar and thoracolumbar fusion: preliminary results. Eur Spine J. 2012;21(suppl 1):S37-S42.

5. Deukmedjian AR, Le TV, Baaj AA, et al. Anterior longitudinal ligament release using the minimally invasive lateral retroperitoneal transpsoas approach: a cadaveric feasibility study and report of 4 clinical cases. J Neurosurg Spine. 2012; 17(6):530-539.

6. Kotwal S, Kawaguchi S, Lebl D, et al. Minimally invasive lateral lumbar interbody fusion: clinical and radiographic outcome at a minimum 2-year follow-up. J Spinal Disord Tech. 2015;28(4):119-125.

7. Moller DJ, Slimack NP, Acosta FL Jr, et al. Minimally invasive lateral lumbar interbody fusion and transpsoas approachrelated morbidity. Neurosurg Focus. 2011;31(4):E4.

8. Isaacs RE, Hyde J, Goodrich JA, et al. A prospective, nonrandomized, multicenter evaluation of extreme lateral interbody fusion for the treatment of adult degenerative scoliosis: perioperative outcomes and complications. Spine (Phila Pa 1976). 2010;35(26)(suppl):S322-S330.

9. Park MS, Deukmedjian AR, Uribe JS. Minimally invasive anterolateral corpectomy for spinal tumors. Neurosurg Clin N Am. 2014;25(2):317-325.

10. Timothy J, Pal D, Akhunbay-Fudge C, et al. Extreme lateral interbody fusion (XLIF) as a treatment for acute spondylodiscitis: Leeds spinal unit experience. J Clin Neurosci. 2019; 59:213-217.

11. Wewel JT, Uribe JS. Retropleural thoracic approach. Neurosurg Clin N Am. 2020;31(1):43-48.

12. Eck JC. Minimally invasive corpectomy and posterior stabilization for lumbar burst fracture. Spine J. 2011;11(9):904-908.

13. Srikantha U, Lokanath YK, Hari A, et al. Minimally invasive lateral transpsoas approach for lumbar corpectomy and stabilization. Surg Neurol Int. 2019;10:153.

14. Smith WD, Dakwar E, Le TV, et al. Minimally invasive surgery for traumatic spinal pathologies: a mini-open, lateral approach in the thoracic and lumbar spine. Spine (Phila Pa 1976). 2010;35(26)(suppl):S338-S346.

15. Sulaiman OAR, Garces J, Mathkour M, et al. Mini-open thoracolumbar corpectomy: perioperative outcomes and hospital cost analysis compared with open corpectomy. World Neurosurg. 2017;99:295-301.

16. Walker CT, Xu DS, Godzik J, et al. Minimally invasive surgery for thoracolumbar spinal trauma. Ann Transl Med. 2018; 6(6):102.

17. Harrop JS, Vaccaro AR, Hurlbert RJ, et al. Intrarater and 
interrater reliability and validity in the assessment of the mechanism of injury and integrity of the posterior ligamentous complex: a novel injury severity scoring system for thoracolumbar injuries. Invited submission from the Joint Section Meeting on Disorders of the Spine and Peripheral Nerves, March 2005. J Neurosurg Spine. 2006;4(2):118-122.

18. Lee JY, Vaccaro AR, Lim MR, et al. Thoracolumbar injury classification and severity score: a new paradigm for the treatment of thoracolumbar spine trauma. J Orthop Sci. 2005; 10(6):671-675.

19. Vaccaro AR, Zeiller SC, Hulbert RJ, et al. The thoracolumbar injury severity score: a proposed treatment algorithm. $J$ Spinal Disord Tech. 2005;18(3):209-215.

20. Chou D, Lu DC. Mini-open transpedicular corpectomies with expandable cage reconstruction. Technical note. J Neurosurg Spine. 2011;14(1):71-77.

21. Chou D, Wang VY, Gupta N. Transpedicular corpectomy with posterior expandable cage placement for L1 burst fracture. J Clin Neurosci. 2009;16(8):1069-1072.

22. Lu DC, Lau D, Lee JG, Chou D. The transpedicular approach compared with the anterior approach: an analysis of 80 thoracolumbar corpectomies. J Neurosurg Spine. 2010;12(6): 583-591.

23. Sasso RC, Renkens K, Hanson D, et al. Unstable thoracolumbar burst fractures: anterior-only versus short-segment posterior fixation. J Spinal Disord Tech. 2006;19(4):242-248.

24. Xu GJ, Li ZJ, Ma JX, et al. Anterior versus posterior approach for treatment of thoracolumbar burst fractures: a meta-analysis. Eur Spine J. 2013;22(10):2176-2183.

25. Fanous AA, Lipinski LJ, Krishna C, et al. The impact of preoperative angiographic identification of the artery of Adamkiewicz on surgical decision making in patients undergoing thoracolumbar corpectomy. Spine (Phila Pa 1976). 2015; 40(15):1194-1199.
26. Uribe JS, Dakwar E, Cardona RF, Vale FL. Minimally invasive lateral retropleural thoracolumbar approach: cadaveric feasibility study and report of 4 clinical cases. Neurosurgery. 2011;68(1)(Suppl Operative):32-39.

27. Park P, Garton HJ, Gala VC, et al. Adjacent segment disease after lumbar or lumbosacral fusion: review of the literature. Spine (Phila Pa 1976). 2004;29(17):1938-1944.

28. Baaj AA, Dakwar E, Le TV, et al. Complications of the miniopen anterolateral approach to the thoracolumbar spine. $J$ Clin Neurosci. 2012;19(9):1265-1267.

\section{Disclosures}

The authors report no conflict of interest concerning the materials or methods used in this study or the findings specified in this paper.

\section{Author Contributions}

Conception and design: Podet, Morrow, Robichaux, DiGiorgio, Tender. Acquisition of data: Podet, Morrow, Robichaux, Tender. Analysis and interpretation of data: Podet, Morrow, Robichaux, Shields, DiGiorgio. Drafting the article: Podet, DiGiorgio, Tender. Critically revising the article: Podet, Shields, DiGiorgio, Tender. Reviewed submitted version of manuscript: all authors. Approved the final version of the manuscript on behalf of all authors: Podet. Statistical analysis: Shields. Administrative/technical/material support: Tender. Study supervision: Tender.

\section{Correspondence}

Adam G. Podet: Louisiana State University Health Sciences Center, New Orleans, LA. apodet@lsuhsc.edu. 